Review

\title{
Porcine ear necrosis
}

\author{
Mateusz Malik $^{\mathrm{a}, *}$, Koen Chiers $^{\mathrm{b}}$, Filip Boyen ${ }^{\mathrm{b}}$, Siska Croubels ${ }^{\mathrm{c}}$, Dominiek Maes ${ }^{\mathrm{a}}$ \\ a Department of Reproduction, Obstetrics and Herd Health, Faculty of Veterinary Medicine, Ghent University, Merelbeke 9820, Belgium \\ ${ }^{\mathrm{b}}$ Department of Pathology, Bacteriology and Avian Diseases, Faculty of Veterinary Medicine, Ghent University, Merelbeke 9820, Belgium \\ ${ }^{\mathrm{c}}$ Department of Pharmacology, Toxicology and Biochemistry, Faculty of Veterinary Medicine, Ghent University, Merelbeke 9820, Belgium
}

\section{A R T I C L E I N F O}

\section{Keywords:}

Animals

Detoxifier

Ear necrosis

Mycotoxins

Pigs

\begin{abstract}
A B S T R A C T
Porcine ear necrosis (PEN) is a condition that mainly occurs in intensive pig production systems and mostly affects piglets after weaning. The syndrome manifests itself with lesions on the pinna, which can heal or become more severe resulting in partial loss of the ear. The pathogenesis of the condition is not fully known. Three different hypotheses for the development of PEN are described in this review: (1) damage of the epidermis due to Staphylococcal exfoliative toxins; (2) occlusion of small blood vessels; and (3) ear biting with subsequent $\beta$-hemolytic streptococcal infection. Risk factors have not been completely elucidated, but viral and bacterial infections, and husbandry factors such as environment, housing conditions and management, have been suggested. It is also possible that some cases are due to a combination of these factors. The role of parasitic infestations has been not investigated. Due to bacterial involvement, severely affected pigs can be treated with antimicrobials. Control and preventive measures should focus on reducing potential risk factors by implementing herd immunization, as well as improvement of sanitary conditions, feed quality (with respect to mycotoxin contamination), management (appropriate stocking density), and environmental conditions (e.g. number of drinkers and feeders and/or optimal ventilation). Further research is needed to better understand the precise etiology and pathogenesis of PEN, so that risk factors can be identified and more targeted control measures can be implemented.
\end{abstract}

(C) 2021 Elsevier Ltd. All rights reserved.

\section{Introduction}

Porcine ear necrosis (PEN), also known as ear tip necrosis, ear necrosis syndrome, ulcerative spirochetosis of the ear, or Streptococcal auricular dermatitis, is characterized by necrotizing ulcerative lesions on the pinna (Richardson et al., 1984). Trauma and bleeding may also be present, which is often associated with trauma and/or ear biting accompanying the necrosis (Park, 2011; Cameron, 2012). Loss of a part of the ear or the entire ear is possible after healing. This is an increasing problem in countries with intensive pig farming (Papatsiros, 2011), but PEN remains a mysterious and unexplored problem in pig production. This review aims to summarize and discuss the current knowledge and to elucidate avenues for future research. The published literature on this topic is limited and must be carefully interpreted, as some papers mention ear lesions and do not specify the cause as ear necrosis or ear biting. In preparing this review, electronic searches were performed in Google Scholar, PubMed, NCBI, ResearchGate,

\footnotetext{
* Corresponding author.

E-mail address: Mateusz.Malik@UGent.be (M. Malik).
}

BioMed Central, and Web of Science, using the keywords 'pig', 'swine', 'porcine', 'piglets', 'ear', 'lesions', and 'necrosis'.

\section{Prevalence}

One Danish study of 90 herds and over 150,000 finisher pigs reported the prevalence of all clinical signs of illness occurring on pig farms. Ear necrosis was by far the most frequently observed clinical sign at $30 \%$. The prevalence of other conditions such as lameness, other skin diseases (superficial abscesses, wounds on the flank), respiratory signs (coughing, forced respiration) or tail bite lesions did not exceed 15\% (Petersen et al., 2008). Another Danish study (Busch et al., 2008) reported that the prevalence of PEN was 46\%. Van Staaveren et al. (2018) reported that ear and tail lesions were among the most common problems (prevalence, 9\%) in an investigation of animal welfare outcomes on 31 Irish farms, representing $12 \%$ of the pig farms in Ireland. Pringle et al. (2009) found the highest prevalence of PEN during the winter (50-70\%) in two organic fattening farms that were monitored over a 2-year period. This indicates that prevalence may vary between countries, between farms, and also over time within a farm. Visible lesions of PEN start to appear mostly in weaned piglets between the 6th and 8th week of life (Papatsiros, 2011; Malik et al., 2020) and may 
remain visible until 14-16 weeks of age (Park, 2011). The average age at which lesions occur is 7 weeks, and initial mild lesions may become severe over an average of 4 weeks (Park et al., 2013).

\section{Diagnosis and scoring of severity of lesions}

Diagnosis is based mostly on the presence of lesions of the affected ears; histologic examination may help to distinguish necrosis from trauma. Identification of the possible cause in an individual herd can be difficult and time-consuming. By minimizing or eliminating potential risk factors, subsequent effects on prevalence and/or severity of PEN can be monitored. Lesion severity can be scored and classified (Pejsak et al., 2011; Malik et al., 2020). Malik et al. (2020) used the following score: score 1, small crust on ear tip; score 2, small wound on ear tip with reddening around; and score 3 , bloody, necrotic wound on ear edge; score 4, partial lack of auricle with necrotic edge. Pejsak et al. (2011) scored severity according to the affected surface of the ear as follows: weak changes covering less than $5 \%$ of the ear surface; mild lesions covering $5-10 \%$ of the ear; and serious lesions covering more than $10 \%$ of the ear. However, a universal scoring method has not yet been established. Scoring methods to describe disease severity often use numerical values to facilitate subsequent data analyses. These scores may not always reflect precisely the severity of the disease or lesion, especially when assessment is by visual appraisal and/or performed by an inexperienced person. A binary score (lesion present or not) is the easiest and potentially the least biased method, but the severity of the lesions is not taken into account, failing to capture important information. More complex scoring systems are more difficult to implement in practice. Other conditions that can change the appearance of the pinna should also be considered e.g. frostbite due to exposure to low ambient temperatures, blue discoloration of the ear caused by systemic infections with pathogens such as porcine reproductive and respiratory syndrome virus (PRRSV), Erysipelothrix rhusiopathiae, Salmonella species (spp), and bovine viral diarrhea virus (BVDV).

\section{Pathogenesis}

The pathogenesis of PEN is not fully elucidated (Richardson et al., 1984) and has not yet been reproduced experimentally. Three hypotheses have been suggested.

According to the first hypothesis, necrosis starts on the outer surface of the injured skin and is caused by exfoliative staphylococcal toxins that damage the epidermis (Park et al.,
2013) by damaging desmosomal cadherins (Bukowski et al., 2010). Fudaba et al. (2005) demonstrated that when exfoliative toxins (ExhA, ExhB, ExhC and ExhD), which coding sequences are present in the genome of Staphylococcus hyicus (S. hyicus) were injected into porcine skin, they caused superficial formation of crusts and blisters, and digested porcine desmoglein 1 (Dsg1). Dsg1 is a desmosome component that binds vertebrate epithelial cells. Similar to S. hyicus toxins (Exh), exfoliative toxins A, B and D (ETA, ETB, ETD) produced by Staphylococcus aureus (S. aureus) belong to serine proteases, but cleave bonds in human Dsg1, causing complex skin infections with blister formation such as staphylococcal scales skin syndrome (SSSS) or bullous impetigo (BI; Nishifuji et al., 2008).

A second hypothesis states that ear necrosis is due to the occlusion of small blood vessels. Pejsak et al. (2011) suggested that Mycoplasma suis (M. suis) infection can lead to the production of cold agglutinins which act as autoantibodies against antigens present on the erythrocyte surface. Together with erythrocytes, they form immune complexes which occlude circulation (Hoelzle et al., 2006). As the ear tips are supplied by small vessels, they are particularly vulnerable to vascular occlusion and subsequent necrosis (Park, 2011). Septic vasculitis leading to necrosis localized to the pinna has been described in dogs and cats, but not yet in pigs (Lee Gross et al., 2005).

The third hypothesis states that trauma e.g. ear biting or environmental factors, is the primary trigger. The injured ear tip becomes infected with $\beta$-hemolytic streptococci present in the mouths of biting pigs and may cause cellulitis and necrosis (Park et al., 2013). It is important to realize that bacteria such as S. hyicus or Streptococcus spp. are part of the porcine skin microbiota, and therefore their presence on the ear will not necessarily lead to PEN. However, if there is skin trauma or tissue damage, these bacteria could multiply and exacerbate the lesions.

\section{Clinical signs}

Porcine ear necrosis lesions vary greatly, ranging from mild to severe (Fig.1). Mild lesions consist of an encrusted sore, localized on the ear tip or ventral margin of the ear. It is unclear why in some cases the ear tip is affected, whereas in others mainly the ventral margin of the ear is affected. Based on the authors' experience, mild cases do not require treatment and can heal. Lesions can also progress to the severe form with epidermal ulceration and necrotic lesions (Richardson et al., 1984). Affected parts of the pinna become dark-red, moist and crusted. The lesions can be present on one or both ears. The effects on pig performance are low, although
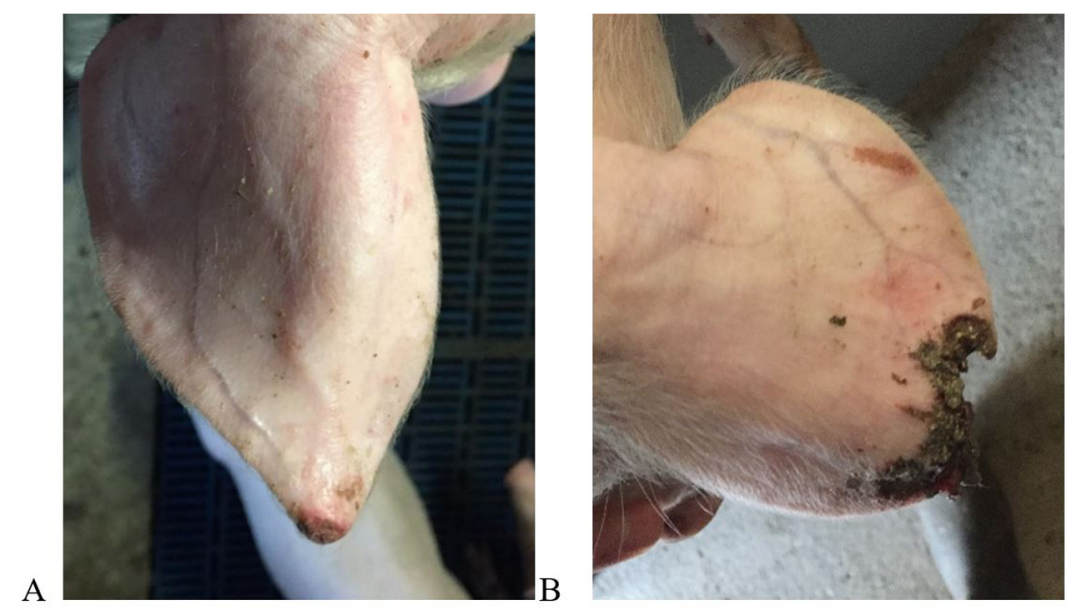

Fig. 1. Graphic presentation of porcine ear necrosis (PEN) lesions: mild lesion (A), severe lesion (B). Photographs taken by Dries Donkers. 
Table 1

Overview of different infectious and non-infectious risk factors for porcine ear necrosis (PEN).

\begin{tabular}{|c|c|c|c|c|c|}
\hline & Risk factors & $\begin{array}{l}\text { Related to } \\
\text { hypothesis a }\end{array}$ & References & Prevention & $\begin{array}{l}\text { Possible effect on } \\
\text { PEN }\end{array}$ \\
\hline \multirow[t]{4}{*}{$\begin{array}{l}\text { Infectious } \\
\text { factors }\end{array}$} & $\begin{array}{l}\text { Immunosuppression caused by } \\
\text { PCV2 and PRRSV }\end{array}$ & 1,3 & Pejsak et al. (2011) & Vaccination & Indirect \\
\hline & Staphylococcus hyicus & 1 & Richardson et al. (1984) & Adequate pen hygiene & Direct \\
\hline & Staphylococcus aureus & 1 & Park (2011) & & Direct \\
\hline & Mycoplasma suis & 2 & $\begin{array}{l}\text { Truszczyński and } \\
\text { Pejsak (2009) }\end{array}$ & $\begin{array}{l}\text { Quick diagnosis and treatment; no commercial } \\
\text { vaccine available }\end{array}$ & Direct \\
\hline \multirow[t]{6}{*}{$\begin{array}{l}\text { Non-infectious } \\
\text { factors }\end{array}$} & $\begin{array}{l}\text { High humidity/poor air quality in } \\
\text { the pen }\end{array}$ & 3 & Smulders et al. (2008) & Adequate ventilation & Indirect \\
\hline & Fully slatted floor without straw & 3 & & Adequate pen design, and additional straw & Indirect \\
\hline & $\begin{array}{l}\text { Low availability of drinkers and } \\
\text { feeders }\end{array}$ & 3 & & & Indirect \\
\hline & High stocking density & 3 & Park et al. (2013) & Appropriate stocking density & Indirect \\
\hline & Mycotoxin contamination & 1 & & $\begin{array}{l}\text { Non-contaminated feed; mycotoxin binder and/ } \\
\text { or detoxifiers }\end{array}$ & Direct \\
\hline & High environmental temperature & 3 & & $\begin{array}{l}\text { Appropriate temperature adjustment/ } \\
\text { ventilation }\end{array}$ & Indirect \\
\hline
\end{tabular}

PCV2, Porcine circovirus type 2; PRRSV, Porcine reproductive and respiratory syndrome virus.

a Three hypotheses, as described in the text: (1) skin damage via toxins; (2) occlusion of blood vessels in ear; and (3) ear biting.

severe lesions might decrease performance and impede the sale of piglets (Park et al., 2013). The most frequent histopathological findings in the mild form are hyperkeratosis, acanthosis and intraepidermal abscesses. The epidermis may be covered by a layer of necrotic cells, degenerated neutrophils, and exudate. Vacuolar degeneration and necrosis of basal cells, with subsequent formation of intra-epidermal vesicles, is less common. Mononuclear cell infiltrates often surround dermal capillaries (Richardson et al., 1984). Histologically, mild PEN is characterized by intraepidermal abscesses, intracellular edema of keratinocytes, parakeratotic hyperkeratosis of the stratum corneum, and/or infiltration of neutrophils (Mirt, 1999). These findings agree with the definition of skin necrosis. ${ }^{1}$ Reiner et al. (2019) also described inflammation and crusts on the ear base of suckling piglets, which did not resemble typical PEN. However, a possible association with PEN, which usually occurs later, in weaned pigs, is worthy of investigation.

\section{Risk factors}

Because the precise etiology and pathogenesis of PEN is largely unknown, many potential risk factors have been suggested to explain lesion prevalence and/or severity. It is generally accepted that PEN is a multifactorial condition in which infectious and noninfectious factors may play an important role (Park, 2011). Table 1 presents an overview of reported risk factors.

\section{Infectious factors}

Infectious factors that may increase the risk for PEN mainly include viral and bacterial infections. Viruses potentially involved include porcine circovirus type 2 (PCV2) and PRRSV. Both viruses are thought to exert immunosuppressive effects, potentially associated with PEN (Pejsak et al., 2011). Tomasini (2015) reported that in immunosuppressed humans, such as patients treated with high-dose corticosteroids, organ transplant recipients or human immunodeficiency virus infected patients, avirulent or lowvirulence bacterial infections can cause septic vasculitis, which

\footnotetext{
1 See: National Toxicology Program, US Department of Health and Human Service, NTP Nonneoplastic Lesion Atlas, Skin - Necrosis. https://ntp.niehs.nih.gov/ $\mathrm{nnl} /$ integumentary/skin/necrosis/skin-necrosis_508.pdf(Accessed 12 March 2021).
}

can theoretically lead to skin necrosis. However, this immunosuppression is likely to be much more pronounced than that caused by PCV2 or PRRSV in pigs. The most common PCV2-associated syndromes are postweaning multisystemic wasting syndrome (PMWS) and porcine dermatitis and nephropathy syndrome (PDNS). Systemic necrotizing vasculitis, as observed in PDNS, can also lead to necrotic skin lesions; however, these vascular lesions can be also observed in the kidneys, spleen or mesenterium (Segalés et al., 2005). The underlying mechanism is thought to be associated with Type III hypersensitivity and deposition of antigenantibody aggregates (Drolet, 2012). Pejsak et al. (2011) demonstrated that vaccination of sows against PCV2 before farrowing decreased the prevalence of PEN in weaned piglets on a PCV2positive farm. Lesion prevalence decreased from $13.1 \%$ to $5.9 \%$ and lesion severity was reduced. When the vaccination protocol ceased, the number of affected piglets rose back to pre-vaccination levels (11.6\%) within 3 months. The percentage of mild and severe lesions was 3-4 times higher in pigs from unvaccinated sows than vaccinated sows. This study indirectly showed the importance of PCV2 in the development of PEN on that individual farm. To the authors' knowledge, no similar studies have been published for PRRSV. The possible role of PRRSV in PEN is based on the frequent prevalence of the virus in weaned pigs and the immunosuppressive characteristics of the virus (Drew, 2000). Therefore, viral caused immunosuppression could allow normal skin microbiota to multiply and cause damage. Risk factors should be distinguished from etiologic factors, as their presence increase the risk for disease, but do not necessarily induce the disease in a specific farm (Thrusfield and Christley, 2018). For instance, on PCV2-free farms, PCV2 infections do not contribute to the problem; similarly, PCV2 vaccination regimens also reduce the likelihood that PCV2 is involved in PEN.

S. hyicus is the most frequently isolated bacterium from ear tip necrosis lesions and can produce toxins that damage the skin (Tanabe et al., 1996). S. hyicus is present at the early stage of ear tip necrosis, but streptococci can also be found in more advanced lesions. Bacterial colonization of the lesions is considered as a crucial step in the breakdown of the epidermis and lesion deterioration (Richardson et al., 1984). Similar to S. hyicus, S. aureus is also commonly found on the skin surface of healthy pigs and can produce toxins that could damage the skin. Therefore, the possible involvement of $S$. aureus in PEN has been suggested (Park, 2011). Park et al. (2013) investigated the causative potential of 
staphylococci. The recovery rate of S. hyicus from 96 ear tissue biopsies originating from 11 different farms was $66 \%$ and the recovery rate of $S$. aureus was $91 \%$, indicating their high prevalence.

Other bacteria mentioned in the literature in association with PEN are spirochetes of the genus Treponema. These are commonly found in skin lesions and mouth microbiota of several species such pigs, cats, dogs and humans. Treponema spp. isolated from porcine gingival and ear lesions are closely related but not identical (Pringle et al., 2009), which brings into question connections between Treponema spp. and ear biting lesions. Park et al. (2013) demonstrated sparse numbers of spirochetes in PEN lesions on histological examination, but could not culture the bacteria. Treponema pedis (T. pedis) is a common species found in porcine ear lesions and shoulder ulcers. Karlsson et al. (2013) reported that spirochetes were found in $73 \%$ of shoulder ulcers, in $53 \%$ of PEN cases and in $9.7 \%$ of gingiva. However, experimental intradermal inoculation of T. pedis did not result in PEN (Karlsson et al., 2017). This may suggest that Treponema might act as secondary agents, but the paper does not rule out their importance as a primary agent.

M. suis is another agent that has been associated with PEN. This bacterium destroys erythrocytes, leading to anemia and bilirubinemia. A subsequent autoimmune response is considered to be important in the pathogenesis in PEN, as cold agglutinins (autoantibodies) can be produced, targeting antigens present on red blood cells surface (Truszczyński and Pejsak, 2009). When body temperature drops, IgM antibodies lead to erythrocyte agglutination (Schmidt et al., 1992). This can take place on the pinna, occluding small vessels and consequently causing ischemia and necrosis of the surrounding tissues. Truszczyński and Pejsak (2009) reported that $M$. suis infection may also cause immunosuppression in the acute phase, rendering pigs more susceptible to other infections. A positive clinical response to appropriate antibiotic therapy confirms the role of bacteria in the development and/or progress of the lesions (Richardson et al., 1984), but this does not prove that bacteria are the primary cause. Alternatively, non-response to antimicrobial therapy cannot exclude an infectious cause, as acquired antimicrobial resistance to commonly used antibiotics can occur (Park, 2011), or inappropriate antimicrobial choices could have been made. The role of parasitic infestations has been not been investigated, however Mirt (1999) mentioned scabies as a possible factor.

\section{Non-infectious factors}

Non-infectious factors potentially involved in PEN include environmental factors such as a fully slatted floor with no straw, poor air quality and high pen humidity (Park et al., 2013), as well as management factors such as high stocking density and early weaning, inadequate availability of drinkers and feeders per pig, and mycotoxin contamination of the feed (Park et al., 2013). Park et al. (2013) also suggested fighting and ear biting as possible factors. Smulders et al. (2008) described four factors which increased ear and tail biting: (1) inadequate number of feeding places; (2) high stable temperature; (3) high ratio of slatted areas; and (4) dry feeding. Camerlink et al. (2015) suggested genetics as an important factor influencing the behavior and biting frequency of pigs. All of these factors may greatly influence animal welfare. Diana et al. (2019) showed also that management factors may impact the development of ear, tail, or skin lesions.

Regarding feed quality, special attention has been paid to mycotoxins. Although there is no published evidence of direct involvement of mycotoxins in PEN, some toxins are considered a risk factor because of their potential immunosuppressive and dermonecrotic effects (Osweiler, 2006). Immunosuppressive effects of aflatoxins, ochratoxins, or trichothecenes have been demonstrated in vitro and in vivo, and can result from depressed T/ B lymphocyte activity, suppressed production of immunoglobulins and antibodies, or decreased complement activity (Corrier, 1991). Weissenbacher-Lang et al. (2012) demonstrated a correlation between high deoxynivalenol concentrations $(0.251 \mathrm{mg} / \mathrm{kg})$ in the feed and microscopic alternations in early PEN i.e. focal epidermal necrosis, histiocyte infiltration, or bacterial growth in the superficial cell debris. Other microscopic lesions, such as collagen lysis, acute vasculitis, granulation tissue, hyperkeratosis, or histiocyte infiltration have been associated with higher concentrations of the ergot alkaloid mycotoxins ergotamine, ergocryptine and ergocristine. Gangrenous ergotism caused by ergot alkaloids is usually a result of vasoconstriction and endothelial damage, which leads to ischemia and finally dry gangrene (Osweiler, 2006).

Other non-infectious factors besides feed contamination with mycotoxins could have an indirect effect on PEN by increasing stress levels and/or aggressive behavior such as ear biting. In this sense, they fit within the third hypothesis of PEN pathogenesis. Further information about possible causes of aggression and biting in pigs can be found in the appropriate in welfare related literature.

\section{Treatment and prevention}

Severely affected pigs should be separated from the pen mates and housed in a hospital pen, in order to prevent biting by other animals. Administration of antimicrobials can slow lesion progression, but severely affected necrotic tissue will not heal using antimicrobial treatment alone, and usually dies. Bacteriological culture should be performed from the lesions and antimicrobial susceptibility testing should be requested. Skin samples should be taken from the transition between healthy and affected tissue beneath crusts, or deep swabs of the lesions. Pejsak and Truszczyński (2009) suggested that the entire age group should be medicated for 2 weeks with amoxicillin or amoxicillin with clavulanic acid. However, oral medication for an extended period does not align with current policy for the prudent use of antimicrobials and reducing the spread of antimicrobial resistance in veterinary medicine (Magnusson et al., 2019). Hansen and Busch (2008) isolated S. hyicus in one Danish herd affected by PEN. After antimicrobial susceptibility testing was performed, affected pigs were injected with sulfadoxine $(200 \mathrm{mg} / \mathrm{mL})$ and trimethoprim $(40 \mathrm{mg} / \mathrm{mL})$ for 5 days. The treatment increased the average daily bodyweight gain by $12 \%$, but did not decrease lesion prevalence or severity. The authors speculated that a higher dose or longer course of treatment might have resulted in a positive effect. Diana et al. (2017) also reported that long term antimicrobial use decreased PEN severity; however, pigs were treated for 9 weeks (sulfadiazine-trimethoprim, $14.4 \mathrm{mg} / \mathrm{kg} / \mathrm{d}$ for 5 days/week). Extended and prophylactic medication regimens are not recommended and are prohibited in some countries because they risk the development of antimicrobial resistance.

One study reported that approximately $95 \%$ of $S$. aureus isolates $(n=87)$ in PEN were resistant to penicillin G and ampicillin, and $75 \%$ of the isolates were not susceptible to tetracycline (Park et al., 2013). Trimethoprim-sulfamethoxazole $(0 \%)$, sulfonamide (5\%) and tiamulin (15\%) had the lowest prevalence of antimicrobial resistance. In the same study, antimicrobial susceptibility of S. hyicus isolates was also performed $(n=63)$. More than $80 \%$ of isolates demonstrated resistance to penicillin $\mathrm{G}$, ampicillin and ceftiofur, but there was almost no resistance to trimethoprimsulfamethoxazole $(0 \%)$ and sulphonamide (5\%). In $M$. suis infections, affected pigs could be treated with oxytetracycline.

Vaccination of piglets against PCV2 or PRRSV may reduce the prevalence of ear necrosis. Sow vaccination may also enhance piglet immunity through antigen specific immunoglobulins or lymphocytes in the colostrum (Joisel et al., 2008). 
Reducing potential risk factors such as adequate pen design, avoiding overcrowding, limiting mixing of pigs, optimal ventilation and air quality, and good feed quality ${ }^{2}$ e.g. by aiming to eliminate exposure to mycotoxins in the feed. Post-harvest mycotoxin mitigation strategies such as mycotoxin detoxifiers, including binders (Jouany, 2007), and modifiers containing yeasts (Molnar et al., 2004), or specific enzymes (Duvick et al., 1998), which can be mixed in the feed to reduce exposure, could be considered. Ear biting and aggression between pen mates can be reduced by improving sanitation, and potentially by dietary supplementation with methionine, threonine and tryptophan (Meer et al., 2017).

\section{Conclusions}

Despite a scarcity of data and wide potential for future studies, the published literature and research on PENS is limited. PENS is a common disorder in pigs worldwide, especially in nursery pigs. The exact etiology and pathogenesis are not yet known; this hampers optimal treatment. It is unclear whether reductions in antimicrobial use due to strict regulations will influence the prevalence and severity of PEN. As the current state of knowledge does not allow causative factors to be ruled in or out, control and prevention measures should focus on reducing potential risk factors reported in the published literature, and increasing the immune status of animals. Under field conditions, practitioners should check for potential risk factors and to assess their role in the problem. Histopathological investigations can help to identify whether associated pathology originated on the skin surface or systemically. It is possible that multiple pathogenic mechanisms could be involved, depending on farm conditions; necrosis on the pinna can be a clinical sign of several specific underlying pathways. To identify and quantify the importance of potential risk factors, large multi-farm observational studies are required. As the existing PEN literature is limited, further research is required, in particular studies investigating prevalence, etiology and pathogenesis, to elucidate factors that may decrease risk.

\section{Conflict of interest statement}

None of the authors of this paper has a financial or personal relationship with other people or organisations that could inappropriately influence or bias the content of the paper.

\section{References}

Bukowski, M., Wladyka, B., Dubin, G., 2010. Exfoliative toxins of Staphylococcus aureus. Toxins 2,1148-1165.

Busch, M.E., Dedeurwaerdere, A., Wachmann, H., 2008. The development and the consequences of ear necrosis in one herd. Proceedings of the 20th International Pig Veterinary Society Congress, Durban, South Africa.

Camerlink, I., Ursinus, W.W., Bijma, P., Kemp, B., Bolhuis, J.E., 2015. Indirect genetic effects for growth rate in domestic pigs alter aggressive and manipulative biting behavior. Behavior Genetics 45, 117-126.

Cameron, R., 2012. Integumentary system: skin, hoof, and claw, In: Zimmermann, J. J., Karriker, L.A., Ramirez, A., Schwartz, K.J., Stevenson, G.W. (Eds.), Diseases of Swine. tenth edn. Willey-Blackwell, Ames, Iowa, USA, pp. 256-257.

Corrier, D.E., 1991. Mycotoxicosis: mechanisms of immunosuppression. Veterinary Immunology and Immunopathology 30, 73-87.

Diana, A., Garcia Manzanilla, E., Calderon Diaz, J.A., Leonard, F.C., Boyle, L.A., 2017. Do weaner pigs need in-feed antibiotics to ensure good health and welfare? Public Library of Science One 12, e0185622.

Diana, A., Boyle, L.A., Garcia Manzanilla, E., Leonard, F.C., Calderon Diaz, J.A., 2019. Ear, tail and skin lesions vary according to different production flows in a farrow-to finish pig farm. Porcine Health Management 5.

Drew, T.W., 2000. A review of evidence for immunosuppression due to porcine reproductive and respiratory syndrome virus. Veterinary Research 31, 27-39.

\footnotetext{
2 See: https://www.merckvetmanual.com/ear-disorders/diseases-of-the-pinna/ necrotic-ear-syndrome-in-swine (Accessed 12 March 2021).
}

Drolet, R., 2012. Urinary system, In: Zimmermann, J.J., Karriker, L.A., Ramirez, A., Schwartz, K.J., Stevenson, G.W. (Eds.), Diseases of Swine. tenth edn. WilleyBlackwell, Ames, Iowa, USA p .367.

Duvick, J., Rood, T., Maddox, J., Gilliam, J., 1998. Detoxification of mycotoxins In Planta as a strategy for improving grain quality and disease resistance: identification of fumonisin-degrading microbes from maize. In: Kohmoto, K., Yoder, O.C. (Eds.), Molecular Genetics of Host-Specific Toxins in Plant Disease. Proceedings of the Third Tottori International Symposium Daisen, Tottori, Japan, pp. 369-381.

Fudaba, Y., Nishifuji, K., Andresen, L.O., Yamaguchi, T., Komatsuzawa, H., Amagai, M., Sugai, M., 2005. Staphylococcus hyicus exfoliative toxins selectively digest porcine desmoglein 1. Microbial Pathogenesis 39, 171-176.

Hansen, K.K., Busch, M.E., 2008. Antibiotic treatment as an intervention against ear necrosis in one herd. Proceedings of the 20th International Pig Veterinary Society Congress, Durban, South Africa.

Hoelzle, L.E., Hoelzle, K., Ritzmann, K., Heinritzi, K., Wittenbrink, M.M., 2006. Mycoplasma suis antigens recognized during humoral immune response in experimentally infected pigs. Clinical and Vaccine Immunology 13, 116-122.

Joisel, F., Charreyre, C., Coulombe, L., Noël, J.C., Longo, S., 2008. Vaccination of sows and gilts against PCV2 diseases: field experiences in Europe. Advances in Pork Production 19, 183-195.

Jouany, J.P., 2007. Methods for preventing, decontaminating and minimizing the toxicity of mycotoxins in feeds. Animal Feed Science and Technology 137, 342-362.

Karlsson, F., Svartström, O., Belák, K., Fellström, C., Pringle, M., 2013. Occurrence of Treponema spp. in porcine skin ulcers and gingiva. Veterinary Microbiology 165, 402-409.

Karlsson, F., Rosander, A., Fellström, C., Backhans, A., 2017. Experimental inoculation of Treponema pedis T A4 failed to induce ear necrosis in pigs. Porcine Health Management 3.

Lee Gross, T., Ihrke, P.J., Walder, E.J., Affolter, V.K., 2005. Vascular diseases of the dermis, Skin Diseases of the Dog and Cat. Clinical and Histopathologic Diagnosis. second edition Blackwell Science Ltd., Oxford, UK p. 238.

Magnusson, U., Sternberg Lewerin, S., Eklund, G., Rozstalnyy, A., 2019. Prudent and efficient use of antimicrobials in pigs and poultry. FAO Animal Production and Health Manual 23. FAO, Rome, Italy, pp. 18-25.

Malik, M., Schoos, A., Chantziaras, I., Biebaut, E., Bernaerdt, E., Beuckelaere, L., Donkers, D., Croubels, S., Maes, D., 2020. Occurrence and impact of ear tip necrosis on daily weight gain in weaned piglets. Proceedings for the International Pig Veterinary Society Congress, Rio De Janeiro Brazil In press.

Meer, Yvd, Gerrits, W.J.J., Jansman, A.J.M., Kemp, B., Bolhuis, J.E., 2017. A link between damaging behaviour in pigs, sanitary conditions, and dietary protein and amino acid supply. Public Library of Science One 12, e0174688.

Mirt, D., 1999. Lesions of so-called flank biting and necrotic ear syndrome in pigs. Veterinary Record 144, 92-96.

Molnar, O., Schatzmayr, G., Fuchs, E., Prillinger, H., 2004. Trichosporon mycotoxinivorans sp. nov., a new yeast species useful in biological detoxification of various mycotoxins. Systematic and Applied Microbiology 27, 661-671.

Nishifuji, K., Sugai, M., Amagai, M., 2008. Staphylococcal exfoliative toxins: "Molecular scissors" of bacteria that attack the cutaneous defense barrier in mammals. Journal of Dermatological Science 49, 21-31.

Osweiler, G.D., 2006. Occurrence of mycotoxins in grains and feeds, In: Straw, B.E., Zimmerman, J.J., D'Allaire, S., Taylor, D.J. (Eds.), Diseases of Swine. ninth edn. Blackwell Publishing Ltd, Oxford, England, pp. 915-929.

Papatsiros, V.G., 2011. Exploration of the connection between Porcine Necrotic Ear Syndrome and PCV2 Infection. Journal of Animal and Veterinary Advances 10, 185-187.

Park, J., 2011. Investigation of Exudative Epidermitis and Ear Necrosis in Pigs. Thesis, Doctor of Veterinary Sciences in Population Medicine. University of Guelph, Ontario, Canada.

Park, J., Friendship, R.M., Poljak, Z., DeLay, J., Slavic, D., Dewey, C.E., 2013. An investigation of ear necrosis in pigs. Canadian Veterinary Journal 54, 491-495.

Pejsak, Z., Truszczyński, M., 2009. Zespół martwicy uszu świń (Porcine ear necrotic syndrome). Życie Weterynaryjne 84, 716-718.

Pejsak, Z., Markowska-Daniel, I., Pomorska-Mól, M., Porowski, M., Kolacz, R., 2011. Ear necrosis reduction in pigs after vaccination against PCV2. Research in Veterinary Science 91, 125-128.

Petersen, H.H., Nielsen, E.O., Hassing, A.-G., Ersbøll, A.K., Nielsen, J.P., 2008. Prevalence of clinical signs of disease in Danish finisher pigs. Veterinary Record 162, 377-382.

Pringle, M., Backhans, A., Otman, F., Sjölund, M., Fellström, C., 2009. Isolation of spirochetes of genus Treponema from pigs with ear necrosis. Veterinary Microbiology 139, 279-283.

Reiner, G., Lechner, M., Eisenack, A., Kallenbach, K., Rau, K., Müller, S., FinkGremmels, J., 2019. Prevalence of an inflammation and necrosis syndrome in suckling piglets. Animal 13, 2007-2017.

Richardson, J.A., Morter, R.L., Rebar, A.H., Olander, H.J., 1984. Lesions of porcine necrotic ear syndrome. Veterinary Pathology 21, 152-157.

Schmidt, P., Kaspers, B., Jüngling, A., Heinritzi, K., Lösch, U., 1992. Isolation of cold agglutinins in Eperythrozoon suis infected pigs. Veterinary Immunology and Immunopathology 31, 195-201.

Segalés, J., Allan, G.M., Domingo, M., 2005. Porcine circovirus disease. Animal Health Research Reviews 6, 119-142.

Smulders, D., Hautekiet, V., Verbeke, G., Geers, R., 2008. Tail and ear biting lesions in pigs: an epidemiological study. Animal Welfare 17, 61-69.

Tanabe, T., Sato, H., Sato, H., Watanabe, K., Hirano, M., Hirose, K., Kurokawa, S., Nakano, K., Saito, H., Maehara, N., 1996. Correlation between occurrence of 
exudative epidermitis and exfoliative toxin-producing ability of Staphylococcus hyicus. Veterinary Microbiology 48, 9-17.

Thrusfield, M., Christley, R., 2018. Observational studies, Veterinary Epidemiology. fourth edn. Wiley, Hoboken, NJ, USA p. 320.

Tomasini, C., 2015. Septic vasculitis and vasculopathy in some infectious emergencies: the perspective of the histopathologist. Giornale italiano di dermatologia e venereologia: organo ufficiale, Societa italiana di dermatologia e Venereologia 150, 73-85.

Truszczyński, M., Pejsak, Z., 2009. Mycoplasma suis and Porcine Eperythrozoonosis including achievements of the last years. Medycyna Weterynaryjna 65, 223227.
Van Staaveren, N., Calderón Diaz, J.A., Garcia Manzanilla, E., Hanlon, A., Boyle, L.A. 2018. Prevalence of welfare outcomes in the weaner and finisher stages of the production cycle on 31 Irish pig farms. Irish Veterinary Journal 71.

Weissenbacher-Lang, C., Voglmayr, T., Waxenecker, F., Hofstetter, U., Weissenböck H., Hoelzle, K., Hoelzle, L.E., Welle, M., Ogris, M., Bruns, G., et al., 2012. Porcine ear necrosis syndrome: a preliminary investigation of putative infectious agents in piglets and mycotoxins in feed. The Veterinary Journal 194, 392-397. 